\title{
Building Better Systems for Learning and Training: Bringing the Entertainment Industry and Simulation Technology Together
}

\author{
William R. Swartout \\ Institute for Creative Technologies, University of Southern California \\ 13273 Fiji Way, Marina del Rey, CA 90265, USA \\ swartoutaict.usc.edu \\ http: //www.ict.usc.edu
}

\begin{abstract}
In 1999, at the University of Southern California the Institute for Creative Technologies (ICT) was established. The ICT was intended to explore a question: what would happen if researchers who understood the technology of simulation and virtual reality worked in close collaboration with people from the entertainment industry who understood how to create compelling stories and engaging characters? What synergies would emerge? Would it be possible to create much more immersive simulation systems for training and learning? In the brief period since the opening of the ICT, we are starting to see the answers to these questions and understand the promise of this approach. In this keynote talk, I will describe some of the insights that have emerged from this collaboration, the major research efforts we have undertaken in areas such as graphics, artificial intelligence and sound, and the integrating virtual reality applications we have produced in areas such as training and leadership development.
\end{abstract}

